\title{
The Surgeon's Limited Responsibility in the Operating Room
}

\author{
Pasquale V. Vairo
}

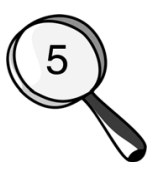

Received: 10 January 2014/ Accepted: 13 January 2014/Published online: 25 February 2014

(C) Springer Science+Business Media New York and International Society of Aesthetic Plastic Surgery 2014

Level of Evidence $V$ This journal requires that authors assign a level of evidence to each article. For a full description of these Evidence-Based Medicine ratings, please refer to the Table of Contents or the online Instructions to Authors www.springer.com/00266.

The recent letter to the editor submitted by Pr. Michel Costagliolo and Dr. Benoit Chaput of the University of Toulouse Rangueil, France presented the interesting question concerning the limits of a surgeon's responsibility in the operating room. The short answer seems to be that the surgeon's responsibility extends to all acts taken; by the surgeon, at the surgeon's direction or under his or her control.

Broadly speaking, where an employee engages the services of a third party in the work of the master, the employee is deemed to be vicariously liable for the torts of that third party under the doctrine of respondeat superior (Restatement, Agency 2d, §362). Thus, when a surgeon, as an employee of a professional corporation, adopts and controls the work of a registered nurse ( $\mathrm{RN}$ ), employed by the hospital, the surgeon as master is vicariously liable for the RN's acts during surgery.

In general, however, an attending surgeon also has the right to rely upon the competency of an OR nurse in the performance of ministerial tasks. So an attending surgeon may not be held vicariously liable for the negligence of a nurse, not in his employ, unless the act giving rise to the injury is one requiring close supervision and instruction. In the 1996 case of Stafford v Molinoff (228 A.D.2d 662), the New York Appellate Court (2nd Dept) affirmed the jury verdict, which found that the defendant surgeon was not responsible for leaving a laparotomy pad in the claimant's abdomen during

P. V. Vairo $(\square)$

Godosky \& Gentile, P.C., 61 Broadway, Suite 2010, New York, NY 10006, USA

e-mail:pvv@godoskygentile.com surgery. At that trial, it was established that as per the hospital's protocol, the OR nurses, and not the surgeons, were responsible for insuring that no foreign objects remained in the patient's body at the end of surgery. Similarly, in the case of Banks v. Barkoukis (231 A.D.2d 598 [2nd Dept 1996]) where an OR nurse inadvertently knocked over an IV pole while adjusting a surgical light, injuring the patient, the surgeon was not responsible since the nurse's task did not require close supervision and instruction by the attending surgeon.

A related issue of apparent or ostensible agency also was recognized as a predicate for malpractice liability by the New York Court of Appeals in the 1986 case of Hill v St. Clare's Hospital [67 N.Y.2nd 72, 499 N.Y.S.2d 904.] In that case, the physician owner of a clinic that was held out to the public as offering medical services, was vicariously liable for the actual treating physician's malpractice at the clinic even though that physician-owner neither controlled, nor took any part in the care and treatment cited by the claimant as improper.

With apparent or ostensible agency, the salient question is whether the patient specifically turned to the hospital for treatment, or simply viewed the hospital as the location where his or her surgeon would operate. One relevant factor to consider in that case is whether the hospital assigned or supplied the surgeon to the patient or whether the surgeon and patient had a pre-existing doctor-patient relationship independent of the hospital.

Ultimately, whether or not a surgeon will be judged liable for the acts of others is fact specific to each individual case and can vary depending on what Operating Room Policies and Procedures are in force at each particular institution and what control the "Captain of the ship" exerts over the surgical staff.

Conflicts of interest The author declares that he has no conflicts of interest to disclose. 